\title{
ОСОБЕННОСТИ ВНЕДРЕНИЯ СОВРЕМЕННЫХ ЦИФРОВЫХ ТЕХНОЛОГИЙ ПРЕДПРИЯТИЯМИ ОБОРОННО-ПРОМЫШЛЕННОГО КОМПЛЕКСА НА ЭТАПЕ РАЗРАБОТКИ ПРОДУКЦИИ ВОЕННОГО НАЗНАЧЕНИЯ
}

\section{THE USE OF MODERN TECHNOLOGIES IN THE DEVELOPMENT OF DIGITAL MODELS OF PRODUCTS AT DEFENSE INDUSTRY ENTERPRISES}

\section{Popkov \\ I. Chistov}

Summary. The article is devoted to the peculiarities of the production of military products that are designed at the enterprises of the defense industry. Taking into account the advanced technologies used in the development and design of products, the authors touched upon the problem of using software resources in creating digital models of products. Attention is paid to the forms of connections of parts used, which eventually form a digital model of the product. The factors influencing the development of more complex digital models are indicated. The authors state that the creation of digital models of products does not entail the involvement of a huge amount of resources, and also makes it possible to reduce the time during which the development of products is carried out, thereby improving the operations associated with the design.

Keywords: digital technologies; product development; digital product model; software resources; design; manufacturing; military-industrial complex.

\author{
Попков Денис Владимирович \\ К.э.н., генеральный директор, $A O$ «ММЗ «Авангард», \\ 2. Москва \\ d_v_popkov@mail.ru \\ чистов Игорь Вадимович \\ Д.э.н., профессор, Военный университет \\ Министерства обороны Российской Федерации, \\ 2. Москва \\ ivchistov@mail.ru
}

Аннотация. В статье рассматриваются этапы жизненного цикла изделий, по номенклатуре относящихся к продукции военного назначения. Учитывая передовые технологии, применяемые в разработке и конструировании изделий, авторами затронута проблема применения программных ресурсов в создании цифровых моделей изделий. Обращено внимание на используемые формы соединений частей, образующих в итоге цифровую модель изделия. Указаны факторы, влияющие на разработку более сложных цифровых моделей. Авторами констатируется, что создание цифровых моделей изделий не влечет за собой вовлечение колоссального объема ресурсов, в то же время позволяет минимизировать время, отведенное на разработку изделий, тем самым совершенствуя операции, связанные с проектированием.

Ключевые слова: цифровые технологии; разработка изделия; цифровая модель изделия; программные ресурсы; конструирование; изготовление; оборонно-промышленный комплекс.
B настоящее время предприятия, входящие в состав оборонно-промышленного комплекса, при выпуске многих изделий, применяют новейшие передовые технологии, а также конструкторские решения [4].

Следует указать, что применение цифровых технологий имеет актуальное значение и играет важную роль среди множества направлений в развитии промышленных предприятий [6].

Применение цифровых технологий революционизирует промышленное производство. Во многих сфе- pax, в том числе и на предприятиях оборонной промышленности данный вид технологий превалирует среди других особенностей, присущих при разработке и производстве продукции военного назначения [1].

При разработке изделий, имеющих отношение к военной продукции, порядок формирования нового образца вооружения, военной и специальной техники включает определенные этапы. В различных источниках наименование этапов трактуется по-разному. Основными этапами жизненного цикла изделия, которые представлены в научной и теоретической литературе являются следующие: 
1. Определение целевой установки изделия, заключающейся в таких составляющих положениях, как:

- предназначение создаваемого изделия (образца вооружения, военной и специальной техники) и сфера его использования;

- проектное выполнение данного изделия;

- его эксплуатационные особенности.

2. Конструирование изделия, включающее:

расчетные мероприятия;

формирование структурных компонентов;

исполнение эскизов, макетов, а также других сопутствующих элементов.

3. Построение процесса создания нового изделия, в который входит:

- установление параметров, характеризующих создаваемое изделие;

- обеспечение средствами, позволяющими изготовить требуемое изделие;

- изготовление экспериментальных моделей изделия.

4. Создание образца изделия, который подлежит апробации для получения сведений о качестве работы изготавливаемого изделия или серии изделий, в том числе коррекция характеристик образца изделия (при необходимости) после наблюдения за его работой.

5. Проверка образца изделия, в составе которой выполняются следующие мероприятия:

- формирование положения, включающего технологию проверки изделия;

- подбор оборудования для проверки;

- установление технических параметров изделия;

- непосредственно проверка работы изделия при влиянии ряда факторов.

6. Организация процесса изготовления нового изделия, включающая:

- обустройство мест изготовления изделий;

- создание вспомогательного оборудования;

- обучение работников, включенных в процесс изготовления изделий.

7. Серийное производство новых изделий.

Безусловно, изготовление образцов вооружения, военной и специальной техники осуществляется работниками оборонных предприятий, имеющих соответствующую профессиональную подготовку [5]. Работники предприятия включены непосредственно в процессы разработки и конструирования новых изделий.

Заметим, что разработка нового изделия начинается с выбора альтернативных предложений, одно из которых признается более подходящим для обоснования изготовления изделия. В итоге разработки создается макет нового изделия. Разработка сравнима с поиском наиболее выгодной и целесообразной методики, включающей приемы, позволяющие выполнить определенную целевую установку при влиянии различного рода факторов. Предложенный макет изделия исследуется, подлежит рассмотрению и внесению изменений (если это потребуется) и признается в качестве образца, с помощью которого производится последующее создание нового изделия.

Можно сказать, что при осуществлении процесса конструирования нового изделия формируется определенная в техническом задании модель изделия. Сконструированная модель представляет собой систему элементов, которые находятся во взаимодействии, как части в целом. Процесс конструирования является той совокупностью операций по созданию изделия, которые выполняются на основании решений, принятых в процессе разработки. В последующем в полученную модель изделия могут вноситься изменения.

Необходимо учесть, что изготовление нового изделия в настоящее время происходит с помощью современных технологий, применяемых в процессе разработки изделий. Традиционное направление разработки изделия, характеризуемое совокупностью операций от момента выстраивания идеи к конструированию изделия и его производству, как правило, представляет собой намеченные планом мероприятия и обоснование действий, входящих в проектную деятельность.

В документах, регулирующих производство военной продукции, содержатся положения, которые необходимо соблюдать соответственно при разработке и производстве изделий [2].

Разрабатывая новое изделие, мы используем, разумеется, определенные средства и оборудование. Средствами, используемыми в разработке изделия, конечно, являются материалы, входящие в дальнейшем в структуру изделия. В итоге разработки изделия появляются спроектированные фигуры, состоящие из элементов, находящихся во взаимосвязи, от которой зависит работа изделия. Эти фигуры существуют в цифровом формате. В них конструктивно отображены все входящие в них части будущего изделия.

Предлагаем обратить внимание на состав цифровой модели изделия, ее характерные свойства в сравнении с настоящим изделием. Одним из главных элементов цифровой модели изделия является деталь. Данная деталь представляет собой часть изделия, имеющая вид геометрической фигуры, параметры определенного элемента в составе сборки изделия. 
Как вы уже поняли, далее пойдет речь о такой категории, как сборка. Что же такое сборка? Сборкой называется сочленение частей изделия, которое выражается в их взаимодействии друг с другом, а также с другими сборками. Кроме того, имеется возможность применения и таких составляющих, как схема, эскиз и прочие категории, учитывая цели и методы разработки изделий.

При организации основных работ, связанных со сборкой изделия, прежде необходимо осуществить мероприятия в конструкторском отделе, которые являются обязательными и выполняются лицами, ответственными за технологичность разрабатываемого изделия [3].

Говоря о цифровой модели изделия в разрезе построения и изучения моделей реально существующих объектов, следует сказать, что, соединяя части в одно целое, мы невольно получаем сведения о структурных элементах изделия. Поэтому, узнав структурные составляющие будущего изделия, можно представить, что существуют разные по структуре формы соединений.

Во-первых, имеется такая форма соединения, называемая сборочной единицей. При данной форме соединения можно обнаружить тождественность структурных элементов реально существующей сборочной единицы.

Во-вторых, можно обнаружить присутствие такой формы соединения, как рабочее. В рабочее соединение входят части изделия, требуемые для разработки специалистом детали или сборочной единицы.

В-третьих, существует соединение частей, при котором они включены в структуру изделия по определенным параметрам (их может быть несколько). Особенность данного вида соединения в том, что таким образом соединенные части не бывают в форме сборочной единицы.

Если рассматривать такие формы соединения, как сборочную единицу и рабочее соединение, учитывая описание этих форм, то при их применении в изготовлении изделия не должно возникать вопросов. Более актуальна форма соединения, при которой части изделия включены в структуру изделия по определенным параметрам. Применяя эту форму соединения, можно обнаружить много способов, позволяющих совершенствовать процесс изготовления изделия.

Напомним, что создание модели изделия в цифровом формате задействует, как правило, большое количество программных средств. В том числе, будут привлечены технические средства электронных вычислительных машин. Это и процессоры, и объемы внешней и оперативной памяти, а также потребуется время на выполнение операций по созданию цифровых моделей изделий.

Совершенствование имеющихся средств электронной вычислительной техники вызывает также и появление новых программных объектов. В итоге происходит привлечение в новое изделие элементов, используемых в виртуальной среде. При развитии компьютерной техники заметно и усложнение самих создаваемых моделей изделия.

При разработке моделей в цифровом формате можно столкнуться с причинами, которые вызывают дополнительную работу в средствах электронной вычислительной техники. К ним относятся:

- трудность построения разрабатываемых цифровых моделей;

- число цифровых моделей.

Трудность построения разрабатываемой модели заключается в проблемах, влияющих на систему и методы разработки, возможности изображения цифровой модели изделия и т.д. Необходимо указать, что существуют различные приемы, используемые для изображения цифровых моделей:

- чистое изображение;

- упрощенное изображение.

Чистое изображение цифровой модели изделия представляет собой тождественное соответствие изделию, которое существует в реальности. В упрощенном же изображении мы имеем возможность получить представление о простых частях, составляющих цифровую модель. В данном случае мы видим только внешние очертания моделей. Изображение в упрощенном виде применяется для частей изделия, не используемых непосредственно в разработке изделия. Этот прием требуется для изображения области, в рамках которой происходят операции по созданию изделия.

Учитывая вышеуказанную систему приемов для передачи изображения цифровых моделей изделий, можно заметить, что это необходимо для обращения внимания на расположенные рядом части изделия и имеющиеся программные ресурсы. Если представить выполнение операций с изделиями, имеющими сложную конфигурацию, то применение упрощенного изображения не окажет должного полезного эффекта в области создания цифровых моделей изделий.

Чтобы претворить в жизнь вопросы, касающиеся эффективности функционирования при разработке 
изделий, представляющих значительное количество соединений частей в составе изделия, применяют деятельность в области сформированных именно для них рабочих соединений, включающих наиболее рациональный и нужный состав элементов.

Трудность в разработке таких изделий состоит в том, что:

- специально для них создается обстановка для разработки;

- изделие технологически представляет объект, состоящий из большого числа соединений.

Это, конечно, влияет на производительность используемых программных средств в электронно-вычислительных машинах. Кроме того, для их разработки требуется колоссальное количество времени. Поэтому, цифровую модель разрабатываемого изделия следует разбивать на необходимое число соединений, учитывая и конструктивно-технологические характеристики, и специфику алгоритма разработки.
Хочется напомнить, что приоритетное значение в данном случае имеет форма соединения частей изделия, при которой они включены в структуру изделия по определенным параметрам. При такой форме соединения части изделия объединены, но не имеются в реальном виде, хотя позволяют создать цифровую модель изделия. Применение вышеуказанных соединений дает возможность минимизировать величину объединенных частей изделия, что также позволяет уменьшить задействованные программные ресурсы путем исключения тех из них, в которых нет необходимости их привлечения.

Таким образом, деятельность по созданию цифровой модели изделия не нуждается в привлечении огромного количества сил и средств, однако дает возможность лицам, принимающим участие в разработке изделия сократить временные параметры, выполняя предварительные действия в разработке, что оптимизирует процессы, связанные с проектированием изделий.

\section{ЛИТЕРАТУРА}

1. Авдеева И.Л., Полянин А.В., Головина Т.А. Цифровизация промышленных экономических систем: проблемы и последствия современных технологий // Известия Саратовского национального исследовательского государственного университета им. Н.Г. Чернышевского. 2019. № 3. С. $238-245$.

2. Баурина С.Б. Проблемы обеспечения качества промышленной продукции в оборонно-промышленном комплексе России // Инновации: перспективы, проблемы, достижения: материалы V международной научно-практической конференции, 17 мая 2017 г., Москва, РЭУ им. Г.В. Плеханова.— Berlin: West-0st-Publishing house, 2017.- - C. 418-423.

3. Журавлев Д.А., Яценко 0.В. Обеспечение точности при создании сложных изделий // Вестник Иркутского государственного технического университета. 2011. № 5 (52). C. 29-33.

4. Закутнев С.Е., Волкова В.В. Критерии и показатели оценки эффективности государственной поддержки деятельности предприятий оборонно-промышленного комплекса // Военный академический журнал.— М., 2017.— № 3 (15). - С. 76-82.

5. Медведев А.В. Проблемы развития кадрового потенциала предприятий оборонно-промышленного комплекса // Вопросы контроля хозяйственной деятельности и финансового аудита, национальной безопасности, системного анализа и управления. Сборник материалов V Всероссийской научно-практической конференции. 2020. С. 113-119.

6. Плотников В.А. Цифровизация производства: теоретическая сущность и перспективы развития в российской экономике // Известия Санкт-Петербургского государственного экономического университета. 2018. № 4 (112). С. 16-24.

○ Попков Денис Владимирович (d_v_popkov@mail.ru ), Чистов Игорь Вадимович ( ivchistov@mail.ru ).

Журнал «Современная наука: актуальные проблемы теории и практики» 